\title{
Correction to: Understanding and mitigating hydrogen embrittlement of steels: a review of experimental, modelling and design progress from atomistic to continuum
}

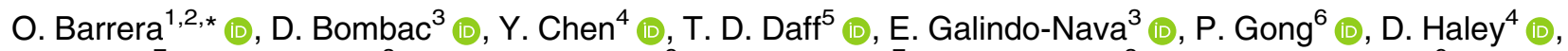

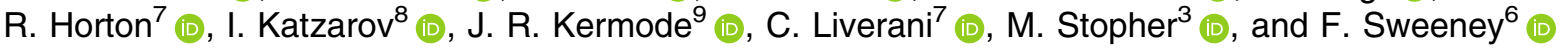

${ }^{1}$ Oxford Brookes University, Wheatley Campus, Wheatley, Oxford OX33 1HX, UK

${ }^{2}$ Department of Engineering Science, University of Oxford, Parks Road, Oxford OX1 3PJ, UK

${ }^{3}$ Department of Materials Science and Metallurgy, University of Cambridge, 27 Charles Babbage Road, Cambridge CB3 OFS, UK

${ }^{4}$ Department of Materials, University of Oxford, Parks Road, Oxford OX1 3PH, UK

${ }^{5}$ Engineering Laboratory, University of Cambridge, Trumpington Street, Cambridge CB2 1PZ, UK

${ }^{6}$ Department of Materials Science and Engineering, University of Sheffield, Mappin Street, Sheffield S1 3JD, UK

${ }^{7}$ Department of Physics, Imperial College London, Prince Consort Road, London SW7 2BB, UK

${ }^{8}$ Department of Physics, King's College London, Strand, London WC2R 2LS, UK

${ }^{9}$ Warwick Centre for Predictive Modelling, School of Engineering, University of Warwick, Coventry CV4 7AL, UK

Published online:

5 April 2018

(C) Springer Science+Business

Media, LLC, part of Springer

Nature 2018

Correction to: J Mater Sci (2018) 53:6251-6290

https://doi.org/10.1007/s10853-017-1978-5

The original paper contains a mistake in the acronym AIDE. The AIDE acronym mistake occurs in the title of the section heading and in the following text on p. 6276, and in the text in the section "Discussion and outlook on the HE mechanisms" on p. 6277. AIDE should read as adsorption-induced dislocation emission.

The original article can be found online at https:/ / doi.org/10.1007/s10853-017-1978-5.

Address correspondence to E-mail: obarrera@brookes.ac.uk 
Also, in several places, words from a paper entitled "Hydrogen embrittlement phenomena and mechanisms" authored by Lynch [1] have been used without being properly quoted. In particular, the sentence on pp. 6276-6277, "For example, dislocations nucleated from crack tips (due to AIDE) may move away from crack tips more readily due to HELP, thereby decreasing the back-stress on subsequent dislocation emission. For crack growth predominantly by AIDE, void nucleation ahead of cracks could be promoted at slip-band intersections by HELP or by HID at particle-matrix interfaces" has been copied from [1]. Moreover, a following sentence on p. 6277, "Increases in back-stress from dislocations emitted due to AIDE can initiate HID, followed by AIDE again when the crack tip had moved away from the stress field of dislocations previously emitted" contains a large number of words used in the original work by Lynch [1] (as do other sentences in this section). We have apologised to Dr. Lynch for using his wordings without acknowledging them.

\section{Reference}

[1] Lynch SP (2012) Hydrogen embrittlement phenomena and mechanisms. Corros Rev 30:105-123. https://doi.org/10.1515/ corrrev-2012-0502 